\title{
Comparative Study of Morphometric Measurements of Placenta in Hypertensive and Normotensive Pregnant Women
}

\author{
MD. Eajaz Ahmed Shariff*
}

Professor, Department of Physiology, Travancore Medical College and Hospital, Thattamala P.O, Kollam, Kerala, India

\author{
DOI: $10.36348 /$ sijap.2019.v02i11.006 \\ | Received: 13.11.2019 | Accepted: 20.11.2019 | Published: 24.11.2019 \\ *Corresponding author: MD. Eajaz Ahmed Shariff
}

\section{Abstract}

Placenta is a vital organ for growth of the fetus. Improper development of the placenta will have a strong impact on the developing fetus resulting in complications like maternal hypertension. The study was aimed to compare the morphometric measurements of placenta in hypertensive and normotensive pregnant women. The study was conducted at Dr BR Ambedkar Medical College and K.C. General Hospital Bangalore. Pregnant women attending antenatal clinics of the hospital regularly and delivered their babies in the same hospital and their placentas were collected. Women who had hypertension in pregnancy after the 20 weeks of gestation, had consistently recorded SBP and DBP of 140 and 90 mm $\mathrm{Hg}$ respectively with or without edema and or proteinuria were included. Placentas delivered were divided into 2 groups. Placentas of 19 hypertensive women were considered hypertensive group and placentas of 24 healthy women were considered Control group. Placental measurements like weight, diameter, thickness, surface area, volume, shape, umbilical cord insertion and membrane attachment of the placenta was assessed using standard methods mentioned in the literature. The study revealed $44.1 \%$ of the patients were hypertensive and $55.8 \%$ of the patients were normotensive respectively. Morphometric measurements of placenta were significantly lower in hypertensive group as compared to the control group $(\mathrm{p}<0.01)$.

Keywords: Hypertensive placentae, normotensive placentae, morphometric measurements, maternal hypertension.

Copyright @ 2019: This is an open-access article distributed under the terms of the Creative Commons Attribution license which permits unrestricted use, distribution, and reproduction in any medium for non-commercial use (NonCommercial, or CC-BY-NC) provided the original author and source are credited.

\section{INTRODUCTION}

Human placenta is defined as hemochorial and deciduate. It is a flattened discoid mass with an approximately oval or circular in outline with an average volume of $500 \mathrm{ml}(200-950 \mathrm{ml})$, average weight about $500 \mathrm{~g}(200-800 \mathrm{~g})$, average diameter $185 \mathrm{~mm}(150$ $200 \mathrm{~mm})$, average thickness $23 \mathrm{~mm}(10-40 \mathrm{~mm})$ and average surface area about $30000 \mathrm{~mm}^{2}$ and thickest at its center[1,2]. Intrauterine presence of the fetus depends on the proper development of the placenta. Improper development of this structure will have a strong influence on the developing fetus resulting in complications like maternal hypertension, gestational diabetes, IUGR etc [3]. Sometimes idiopathic intrauterine growth restricted babies can be the result of pregnancy but there will be no specific complications in that case, placental examination will provide an vision into that specific condition. Hypertension is one of the common complications met with in pregnancy and diagnosed if maternal blood pressure is more than 140/90mmHg.Hypertension in pregnancy may be chronic hypertension (onset before pregnancy) or may be induced due to pregnancy like gestational hypertension, pre-eclampsia and eclampsia. Hypertensive disorders are responsible for 5-8\% of all maternal deaths [4].In hypertension, there will be decrease in the blood supply due to vasoconstriction. Careful examination will provide an accurate account of in utero events of fetus. Prenatal experiences of mother and baby can be diagnosed by the examination of placenta, maternal blood vessels. Many of the complications will be reflected in the gross architecture of the placenta that one minute examination is so important. This will protect doctors from medicolegal issues if there is any poor maternal or fetal outcome and also help them in the management of the successive pregnancies and the infants [5]. Attempt is made to study and compare the morphometric measurements of placenta in hypertensive and normotensive pregnant women.

\section{MATERIALS AND METHODS Study Design \\ This is a hospital based prospective, crossectional study conducted at Dr BR Ambedkar Medical College and K.C.General Hospital Bangalore.}


Forty three pregnant women attending regular antenatal checkups of which 19 were hypertensive (Hypertensive group) and 24 were healthy women (Control group) gave birth to singleton babies; their placentas were collected and studied. Written Informed consent was obtained from all the participants after explaining the study in detail and ensuring the confidentiality. The study protocol was approved by institutional human ethical committee.

Complete medical, social and obstetric history was recorded to identify the confounders. The mothers were examined clinically along with recording of relevant investigation reports. All control subjects had no history of raised blood pressure at any stage during their life and had no evident hypertension and proteinuria during pregnancy. Hypertensive women who had hypertension in pregnancy after the 20 weeks of gestation, had consistently recorded SBP and DBP of 140 and $90 \mathrm{~mm} \mathrm{Hg}$ respectively with or without edema and/or proteinuria were included. Women with twins and any other serious diseases like endocrine disorders, cardio vascular diseases, respiratory diseases, renal diseases. Diabetes mellitus, anemia was excluded from the study.

\section{Placental Measurements}

Placental weight, diameter, thickness, surface area, volume, umbilical cord insertion and membrane attachment of the placenta was assessed using standard methods mentioned in the literature [6]. The placenta with cords and membranes were collected and observed immediately after the delivery. Any abnormality of the umbilical cord and membrane was recorded. In all these cases, the amnion and chorion were trimmed off from the placenta. The umbilical cord was cut at a distance of $10 \mathrm{~cm}$ from the site of insertion. Placentae were washed in slow running tap water dried with the help of blotting paper. The placentae were weighed with a standard weighing machine. Diameter was measured by placing the placenta in a flat tray; the first maximum diameter was measured by a metallic scale graduated in centimeters $(\mathrm{cm})$. Then second maximum diameter was taken at right angles to the first one. The mean of two measurements was considered as the diameter of the placenta expressed in centimeters. The thickness of the placenta was measured by inserting a calibrated Knitting needle at the center of placenta and measured in centimeter each placenta was placed on fetal surface.

The maternal surface area of the placenta was calculated using the following formula: Surface area $=\pi \mathrm{d} 1 \times \mathrm{d} 2 / 4$, (where $\mathrm{dl}$ is the largest diameter and ds are the smallest diameter. Circumference is calculated by formula $\pi \times \mathrm{d}$. Volume is calculated by formula $\pi \mathrm{r} 2 \mathrm{~h}[6]$.

\section{STATISTICAL ANALYSIS}

The data obtained was tabulated and analysed by SPSS programme and Graph Pad Prism version 5.0
.Analysis of Variance (ANOVA) and student' $t$ ' test were used for the analysis. The level of significance of $p$ value $(p<0.01)$ were considered significant.

\section{RESULTS}

Total number of the mothers participated in the study were 43 of which 19 mothers were hypertensive $(44.1 \%)$ and 24 mothers were normotensive (55.81\%). Birthweight (in $\mathrm{Kgs}$ ) of the hypertensive and normotensive mothers were $2.01 \pm 0.4$ and $2.6 \pm 0.3$ respectively [Fig 1]. The study revealed birth weight of the hypertensive mothers were significantly lower than the normotensive mothers $(\mathrm{p}<0.01)$.

Mean Placental weight (in grams) in hypertensive and normotensive mothers were 397.08 89.3 and $485.2 \pm 47.2$ [Fig 2] and Mean placental area (sq. cms) were 201.78 \pm 58.3 and, 245.1 \pm 61.2 [Fig 3] respectively. Morphometric measurements weight and surface area showed significantly $(\mathrm{p}<0.01)$ lower values in hypertensive placentae in comparison to the normotensive placentae. There was no gross significance in the mean placental cotyledons per placenta between the groups, but there was statistically significant difference seen in mean infarcted area and mean calcified area of the placental in hypertensive mothers $(\mathrm{p}<0.01)$ when compared to the normotensive mothers [Table 1]. Marginal insertion of the cord was found to be $4.7 \%$ and $2.1 \%$ in hypertensive and normotensive placentae respectively.

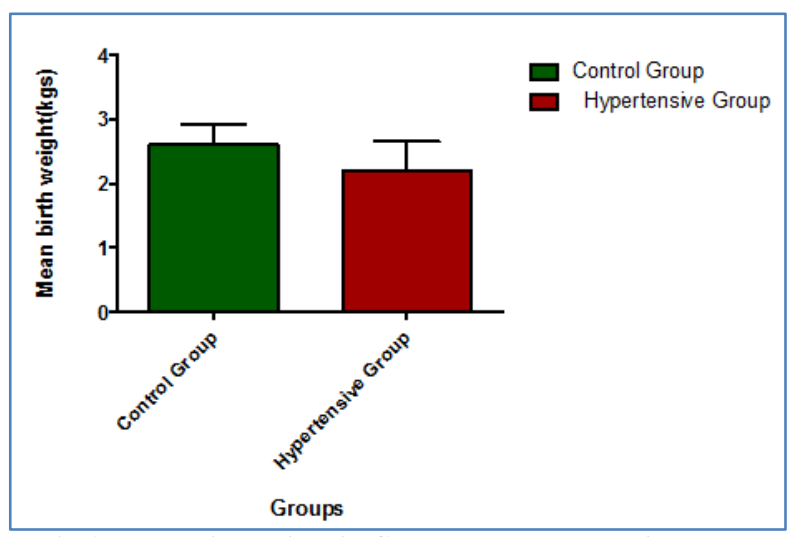

Fig-1: Mean Birthweight in Control and Hypertensive group

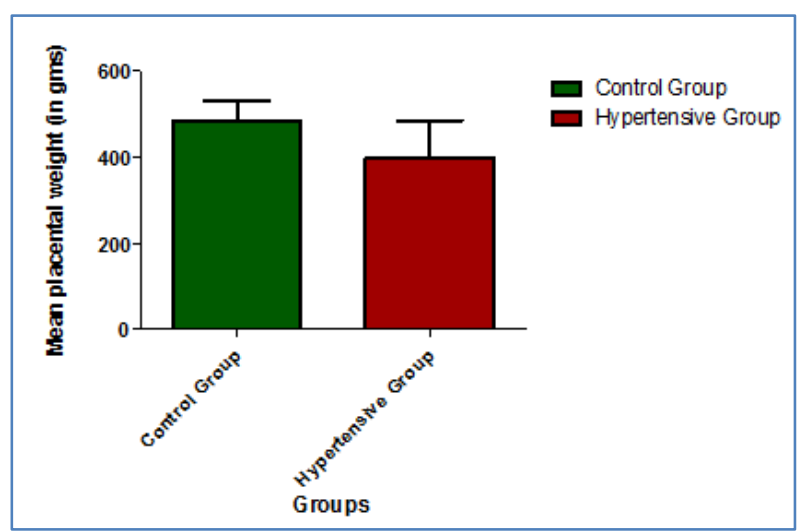

Fig-2: Mean Placental Weight and Hypertensive group 


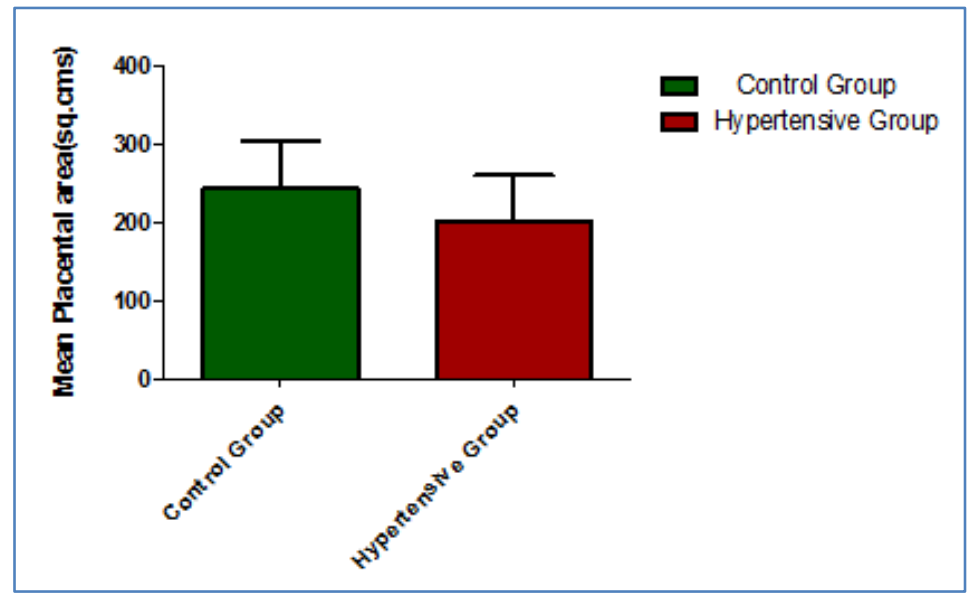

Fig-3: Mean Placental in control and Hypertensive group

Table-1: Mean placental values in control and Hypertensive groups

\begin{tabular}{|l|c|c|}
\hline Parameters & $\begin{array}{c}\text { Control Group } \\
(\mathbf{n = 2 4})\end{array}$ & $\begin{array}{c}\text { Hypertensive Group } \\
(\mathbf{n = 1 9 )}\end{array}$ \\
\hline & Mean \pm SD & Mean \pm SD \\
\hline Cotyledons per Placenta & $16 \pm 2$ & $15 \pm 2$ \\
\hline Placental Volume(cc) & $608.9 \pm 211$ & $369 \pm 79^{*}$ \\
\hline Infarcted area in the Placenta in number & $1.22 \pm 0.2$ & $3.2 \pm 1.0^{*}$ \\
\hline Calcified area in the placentae in number & $1.5 \pm 0.2$ & $2.2 \pm 1.3^{*}$ \\
\hline Marginal insertion of the cord (\%) & $2.1 \pm 0.5$ & $4.7 \pm 1.6^{*}$ \\
\hline
\end{tabular}

*Statistically significant $(\mathrm{p}<0.01)$

\section{DISCUSSION}

Placental weight and birth weight of the neonate are commonly used variables [7]. Our study revealed that birthweight (in $\mathrm{Kgs}$ ) of the hypertensive and normotensive mothers were $2.01 \pm 0.4$ and $2.6 \pm 0.3$ respectively. Birth weight of the hypertensive mothers were significantly lower than the normotensive mothers $(\mathrm{p}<0.01)$. Our findings were in agreement with other studies [8-10].

Mean Placental weight (in grams) in hypertensive and normotensive mothers were 397.08 \pm 89.3 and $485.2 \pm 47.2$ [Fig 2] and Mean placental area (sq. cms) were 201.78 \pm 58.3 and, 245.1 \pm 61.2 [Fig 3] respectively. Morphometric measurements weight and surface area showed significantly $(\mathrm{p}<0.01)$ lower values in hypertensive placentae in comparison to the normotensive placentae. Previous studies have reported that there is decrease in surface area weight of placenta in hypertensive cases in comparison with normotensive cases. Our findings were in agreement with other studies $[11,12]$.

The measurements of the delivered placenta reveal the collective development of the placenta from conception to delivery. Placental weight is one of several standard placental measures by which placental growth can be characterized. Laterally expanding growth of the chorionic plate and ramifications of the villous and vascular nutrient exchange surface, reflected in increased thickness of the chorionic disk [13]. Other studies have shown a linear correlation exists between birthweight of the newborn and placental weight in uncomplicated pregnancies. Mild and severe gestational hypertension a linear relationship exists with placental weight and birthweight of the newborn [14].

We found that there was no gross significance in the mean placental cotyledons per placenta between the groups, but there was statistically significant difference seen in mean infarcted area and mean calcified area of the placental in hypertensive mothers $(p<0.01)$ when compared to the normotensive mothers. Marginal insertion of the cord was found to be $4.7 \%$ and $2.1 \%$ in hypertensive and normotensive placentae respectively. Our findings were in disagreement with other findings [15], who have reported higher incidence of placental infarction in toxemia of pregnancy. Placental calcification was observed more commonly in placentae from hypertensive patients than the normal group in the study. Placental calcification of $73.34 \%$ cases was observed in hypertensive pregnancies [16].

\section{CONCLUSION}

The study concluded that birth weight of the hypertensive mothers were significantly lower than the normotensive mothers. Morphometric measurements weight and surface area showed significantly lower values in hypertensive placentae in comparison to the normotensive placentae. But there was statistically significant difference seen in mean infarcted area and mean calcified area of the placental in hypertensive mothers. Mothers with hypertension during pregnancy have to be carefully monitored and adequate measures 
to be taken prevent the complications of pregnancy induced hypertension.

\section{ACKNOWLEDGEMENTS}

I extend my gratitude and thanks for the Doctors, nursing staff of K.C general hospital Bangalore for all the technical support during the course of the study.

\section{REFERENCES}

1. Gupta, C., Harode, H. A., D'souza, A. S., \& Sharma, A. (2015). A morphological and morphometric study of placenta with its clinical implications. Tropical Journal of Medical Research, 18(2), 85.

2. McMaster, M. T., Zhou, Y., \& Fisher, S. J. (2004, November). Abnormal placentation and the syndrome of preeclampsia. In Seminars in nephrology. WB Saunders, 24(6): 540-547

3. Fisher, S. J. (2004). The placental problem: linking abnormal cytotrophoblast differentiation to the maternal symptoms of preeclampsia. Reproductive Biology and Endocrinology, 2(1), 53.

4. Park, K.(2009). Textbook of Preventive and Social Medicine, $20^{\text {th }}$ edition, Banarsidas Bhanot Publishers, Jabalpur, 482.

5. Ptacek, I., Smith, A., Garrod, A., Bullough, S., Bradley, N., Batra, G., \& Heazell, A. E. (2016). Quantitative assessment of placental morphology may identify specific causes of stillbirth. $B M C$ clinical pathology, 16(1), 1.

6. Akshara, V. R., Ramakrishnan, P. K., Chitra, S., Kumar Sai Sailesh, S. K. S., \& Seema Valsalan, E. (2018). Morphology and the morphometric measurements of hypertensive and normotensive placenta.

7. Akshara, V. R., Ramakrishnan, P. K., Chitra, S., Kumar Sai Sailesh, S. K. S., \& Seema Valsalan, E. (2018). Morphology and the morphometric measurements of hypertensive and normotensive placenta.

8. Damania, K. R., Salvi, V. S., Ratnaparki, S. K., \& Daftari, S. N. (1989). The placenta in hypertensive disorder in pregnancy. $J$ Obst and Gynaecol Ind, 39, 28-31.

9. Kalousek, D. K., \& Langlosis, S. (1994). The effects of placental and somatic chromosomal mosaicism on foetal growth. Ward RHT, Smith SK, Donnai (eds). Early foetal growth and development. RCOG Press, London, 245-56.

10. IPGMER, K. (2005). A study of placenta in normal and hypertensive pregnancies.J. Anat. Soc. India, 54(2), 1-9.

11. Browne, J. M., \& Veall, N. (1953). The maternal placental blood flow in normotensive and hypertensive women. BJOG: An International Journal of Obstetrics \& Gynaecology, 60(2), 141147.

12. Rath, G., Garg, K., Anand, C., \& Kawle, M. (1994). Vascular pattern of human placenta in complicated pregnancy: A corrosive cast study. Ann. NAMS India, 30, 17-22.

13. Shariff, M. E. A., \& Taufiq, F. (2018). Scholars International Journal of Anatomy and Physiology.

14. Udainia, A., \& Jain, M. L. (2001). Morphological study of placenta in pregnancy induced hypertension with its clinical relevance. J Anat Soc India, 50(1), 24-27.

15. Bhatia, A., Sharma, S. D., Jalnawalla, S. F., \& Sagreiya, K. (1981). A comparative study of placental pathology and fetal outcome. Indian journal of pathology \& microbiology, 24(4), 277283.

16. Torpin, R., \& Swain, B. (1966). Placental infarction in 1,000 cases correlated with the clinical findings. American Journal of Obstetrics \& Gynecology, 94(2), 284-285. 